\title{
Star Formation at High Angular Resolution: Summary and Outlook
}

\author{
Hans Zinnecker \\ Astrophysical Institute Potsdam, Germany
}

\begin{abstract}
I give a personal summary of IAU-Symposium 221 "Star Formation at High Angular Resolution", including general impressions and scientific highlights (both observations and theory). Some future directions, like infrared interferometry and advanced SPH numerical simulations, are described. In passing, I also make some critical comments about information overload and the dangers of decreasing information transfer efficiency in the age of power-point.
\end{abstract}

\section{General Impression}

IAU Symposium 221 was the last of the six IAU Symposia held during the IAU General Assembly in Sydney, but not the least. Indeed the theme of this symposium ("Star Formation at High Angular Resolution") was carefully chosen and provided a timely synopsis of where we stand and where we are going.

When I compare my expectations before the symposium with my reflections after the symposium, my overriding impression is the following: this was not just another star formation conference, but the speakers took the focus of the meeting, i.e. the high angular resolution aspect, extremely seriously, and in this sense the symposium set an excellent example. Congratulations to the SOC for steering the invited speakers in the right direction to meet their challenge. What the SOC forgot to tell them is that "a talk is not a paper"-but actually the speakers should know this themselves. With $\sim 60$ talks given, the danger of information overload is high, and in some cases (I won't mention names) the limit was exceeded (too many power-point slides per unit time).

A yet more serious problem surfaced when it became clear that not enough time was set aside to view and to discuss the $\sim 150$ posters. Disregarding the effort that went into the poster preparation caused some frustration, augmented by the announcement that the results of the posters could not be included in the printed proceedings. I understand that a web-based solution has been found to publish the essence of the posters. Most posters were nicely prepared and many new results were reported. I read them all during the night of the conference dinner (sacrificing the dinner!). 


\section{Scientific Highlights}

\subsection{Observations}

Among the observational highlights of the symposium were the first interferometric results from the VLT and Keck long-baseline interferometers, with baselines of $102 \mathrm{~m}$ and $85 \mathrm{~m}$, and at wavelengths of $10 \mu \mathrm{m}$ and $2 \mu \mathrm{m}$, respectively (see the papers presented by Leinert et al. and Akeson et al.). Several new adaptive optics results in the near-infrared from VLT/NACO, Keck, Subaru and GeminiNorth (Hoku'paa) were also reported.

$\mathrm{Mm}$-interferometry is already more advanced and seems to be in full swing: numerous results from the Plateau de Bure (PdBI), Owens Valley/BerkeleyIllinois arrays (OVRO/BIMA), and Nobeyama array (NMA) were presented, especially on protostellar collapse and young stellar object (YSO) disk structure. Disk structure was also investigated with the Hubble Space Telescope (HST), and new direct images (with and without central coronographic masking of the star) were shown.

Finally, I was intrigued by the new VLBA SiO maser observations of the compact radio source "I" in the Orion Kleinmann-Low nebula (Greenhill et al.) and their new implication for the outflow of what is perhaps the nearest massive star in the making. I suppose the extremely high spatial resolution maser observations, while still somewhat underrated today, will come to be properly appreciated in the near future, particularly when we better understand the dynamical information they carry about the dense circumstellar gas (disks and jets).

\subsection{Theory}

One of the highlights in theory were the 3D SPH numerical simulations (movie) of the collapse and turbulent fragmentation of a 50 solar mass protocluster cloud shown by Mathew Bate. Not everyone is fully convinced that the evolution is so fast and violent as in the movie. After all, no replenishment of the initial turbulence and no magnetic fields are included, which would both slow down the dynamics. Some reservations were raised (Philippe Andre) that the model did not produce any pre-stellar cores, as for example observed in the Rho Ophiuchi dark cloud. Bruce Elmegreen suggested (private communication) that switching on self-gravity in Bate's simulation gradually might help to generate these cores.

In contrast, the other highlight was Frank Shu's new analytical model on how to form magnetically super-critical cores embedded in magnetically subcritical envelopes. Such cores are formed from a turbulent velocity field with magnetic fields. Cores of various masses are formed, and the core mass spectrum determines the stellar IMF, as these cores form stars with a constant efficiency $(1 / 3)$ due to feedback from wide-angle $\mathrm{X}$-winds. Whether stellar masses ultimately result from competitive accretion (Bate), following essentially nonmagnetic turbulent fragmentation, or from pre-stellar supercritical cores generated by an MHD turbulent velocity field (Shu), remains a major open question that needs to be resolved. I heard that a method to include magnetic fields into SPH simulations has recently been developed (Monaghan and Price), so we can hope for new progress to resolve this key issue. 
Finally, I'd like to mention the clear review on high-mass star formation via accretion by Harold Yorke, in which he showed that radiation pressure on dust is not only important in spherically symmetric collapse but also $2 \mathrm{D}$ axi-symmetric accretion, and that therefore high-mass star formation by disk accretion is not really a scaled-up version of low-mass star formation by disk accretion, contrary to common belief.

\section{High Spatial Resolution Observations of the Future}

\subsection{Near-Future}

I here define the near future as the time up to the next two IAU General Assemblies (2006 in Prague, 2009 in Rio de Janeiro).

Many great observing opportunities will come with the new interferometric long-baseline devices in the $1-2 \mu \mathrm{m}$ micron and $10-20 \mu \mathrm{m}$ regimes, in particular with regard to the direct detection of giant planets and the spatial resolution of circumstellar disks. A major role will be played by the VLT-interferometer (AMBER and MIDI, respectively) and the Keck I/II interferometric beam combination. Hopefully the LBT interferometer on Mt. Graham in Arizona (two $8.4 \mathrm{~m}$ telescopes on a common mount, $14.4 \mathrm{~m}$ centre-to-centre, maximum baseline $23.8 \mathrm{~m}$ ) will also fulfill its promise as a first class interferometer (with excellent $u v$ coverage and thus, unlike VLTI and Keck-I, good IMAGING capability important for complex objects where a priori knowledge of the source structure is not available). The LBT interferometer can also be used as a nulling interferometer to detect the zodiacal light from extra-solar planetary systems (Roger Angel, in Nature), a pre-requisite for NASA's Terrestrial Planet Finder and ESA's Darwin mission in the far-future.

By 2006, all the new mm interferometers (SMA, NMA and CARMA = OVRO+BIMA, see the comprehensive review by Anneila Sargent) will be fully in operation, and ATCA in Australia will serve the southern hemisphere-where the mm sky at high angular resolution is still unchartered and pristine, i.e. an astronomical goldmine and a most welcome preparation for ALMA in Chile in the far-future. We also must not forget VLBA/VLBI and the extended VLA (EVLA) which will continue to be used to probe circumstellar structure and dynamics (masers and ultracompact HII regions) in unprecedented detail.

And then there will be the harvest from SIRTF (NASA's $85 \mathrm{~cm}$ mid-infrared space telescope, launched $2003 \mathrm{Aug}$ 25) and later HERSCHEL (ESA's $3.5 \mathrm{~m}$ far-infrared space telescope, to be launched 2007), as well as SOFIA (the 747jumbo jet with a $2.5 \mathrm{~m}$ telescope for far-infrared astronomy, a collaboration between USA and Germany). Although limited in absolute angular resolution, these telescopes will provide the best possible diffraction-limited resolution at wavelengths difficult or impossible to access from the ground. Star formation studies are bound to greatly benefit from these facilities, because protostars are cool objects whose spectral energy distribution peaks in the mid-to-far IR.

\subsection{Far-Future}

I define the far future to be the time between 2010 and 2020. During this time, we will see a whole new suite of high spatial resolution facilities, at various wave- 
lengths, including the James Webb Space Telescope (JWST, successor to HST), the Atacama Large Millimetre Array (ALMA, successor to SMA etc.), the Space Infrared Interferometric Telescope (SPIRIT, a 30-50 m space interferometer, successor to HERSCHEL/SOFIA), and the X-ray Evolving Universe Spectrometer (XEUS, successor to CHANDRA). Eagerly awaited, the micro-arcsec astrometry missions GAIA (ESA) and SIM (NASA) will revolutionize positional and proper motion measurements, with far-reaching implications (e.g. detection of unseen planetary companions, probing the $6 \mathrm{D}$ phase space of stars in the Galaxy and elsewhere). And then there may be CELT (the $30 \mathrm{~m}$ California Extremely Large Telescope) and even OWL (ESO's $100 \mathrm{~m}$ Overwhelmingly Large Telescope), with the detection of earth-like planets around other stars as the key science driver. These telescopes may also be suitable to penetrate as much as 200 magnitudes of visual dust absorption, perhaps enough to detect AND resolve the deeply obscured dense protoclusters of massive stars before they hatch.

\section{High Spatial Resolution Numerical Simulations of the Future}

In observations of crowded fields in star clusters there is usually a critical angular resolution depending on the cluster distance. For example, while for the Orion Trapezium cluster 1 arcsec suffices, for NGC 3603 (ten times more distant) we need 0.1 arcsec and for R136 in 30 Dor (100 times more distant than Orion) we need 0.01 arcsec to resolve all the individual stars (cf. Zinnecker 2001). Similarly, there is a "critical" numerical resolution for SPH simulations. In order to resolve the minimum Jeans mass in a protocluster collapse and fragmentation calculation one needs roughly 100 particles, and this mass is typically around a Jupiter mass. Hence, to model a cloud of $X$ solar masses of gas, one needs roughly $10^{5} \times X$ particles. So, $10^{7}$ particles would allow one to do a $100 \mathrm{M}_{\odot}$ cloud, $10^{8}$ a $1000 \mathrm{M}_{\odot}$ cloud, etc., capturing all the fragmentation down to brown dwarfs. The latter is now within reach, for example using the UK Advanced Fluid Facility UKAFF (as Bate and Bonnell do). In the near future, magnetic fields will be included in $\mathrm{SPH}$, as will be radiative transfer. A number of groups are working on their implementation (difficult). In the far future, we may expect to see a hybrid numerical scheme, in which SPH will be combined with adaptive mesh refinement (AMR) techniques, in an effort to resolve collapsing cores and disks in a turbulent hierarchy of structure formation in molecular clouds. However, this may require much more parallel computing than today. The main worry with these highly sophisticated numerical schemes is not the scheme itself, but more likely some missing physics.

How does the CPU time behave as a function of the number of SPH particles? It goes as $N \log N$ due to gravity. This is calculated using a tree structure for which each calculation of gravity goes as $\log N$ and you have to calculate it for each particle, giving the first $N$. For $N$ of the order of $10^{7}$ particles, it takes about half a month of CPU time!

\section{Wise Words and Quotes to Remember}

It may be in order to recall a few of the bonmots that I heard at the symposium. The observers made a good (Australian) start when one of them (Saito) chris- 
tened the nebula that he studied the "Koala Nebula". The theorists quickly followed with a "rectangular protostar" (Hartmann) and a quick back-of-anemail radiative transfer calculation (Yorke). Then Jean Turner remarked that O-stars are nasty from birth to death, and Melvin Hoare compared the search for disks around massive stars to the search for weapons of mass destruction. Kevin Luhman reminded us early on that accuracy is not the same as precision, and Derek Ward-Thompson hastened to add that development is not equal to progress. Leo Woltjer was quoted in absentia by someone with the statement "the higher the ignorance, the stronger is the magnetic field". Finally, this author invented Bart Bok's uncertainty principle "fun times work equals unity" implying that too much fun means no work gets done, while doing too much work provides no fun. With the right balance, Bok's principle suggests our star formation work is good fun - Bok was director at Siding Spring Observatory 1957-1967 (see Gascoigne in the March-April issue of Mercury in 1984, and also Lada \& Reipurth in the same issue).

\section{Next Star Formation Meetings}

The next big conference in our field will be Prototars and Planets V, to be organized by Bo Reipurth in Kona/Hawaii in late October 2005. There is a plan to apply for an IAU-Symposium on "massive star birth" led by Drs. Conti and Churchwell, probably in Sicily in spring 2005. I also suggest to celebrate "50 years of the Salpeter-IMF". It was from the Australian National University in Canberra that Ed Salpeter submitted his famous paper with the first derivation of the empirical stellar IMF in July 1954, which appeared in 1955 in ApJ, 121, 161.

\section{Acknowledgments}

We all owe special thanks to Michael Burton (and his co-chairs Tyler Bourke and Ray Jayawardhana) for bringing so many of us together for this topical symposium (over 2,000 registered participants to the GA). Michael Burton also organised a memorable "Science in the Pub" session with the theme: what is a planet? (with lots of beer). Finally, a tribute to Sydney: Darling Harbour was a spectacular conference venue probably unmatched in the world. I would also like to acknowledge the Deutsche Forschungsgemeinschaft for providing me with travel support to attend the IAU GA.

\section{References}

Zinnecker, H., 2001, The VLT and the Powers of 10: Young Clusters Home and Away. In The Origins of Stars and Planets: the VLT View, ESO Workshop, Garching, April 2001, p179. Eds. J. Alves \& M. McCaughrean. 


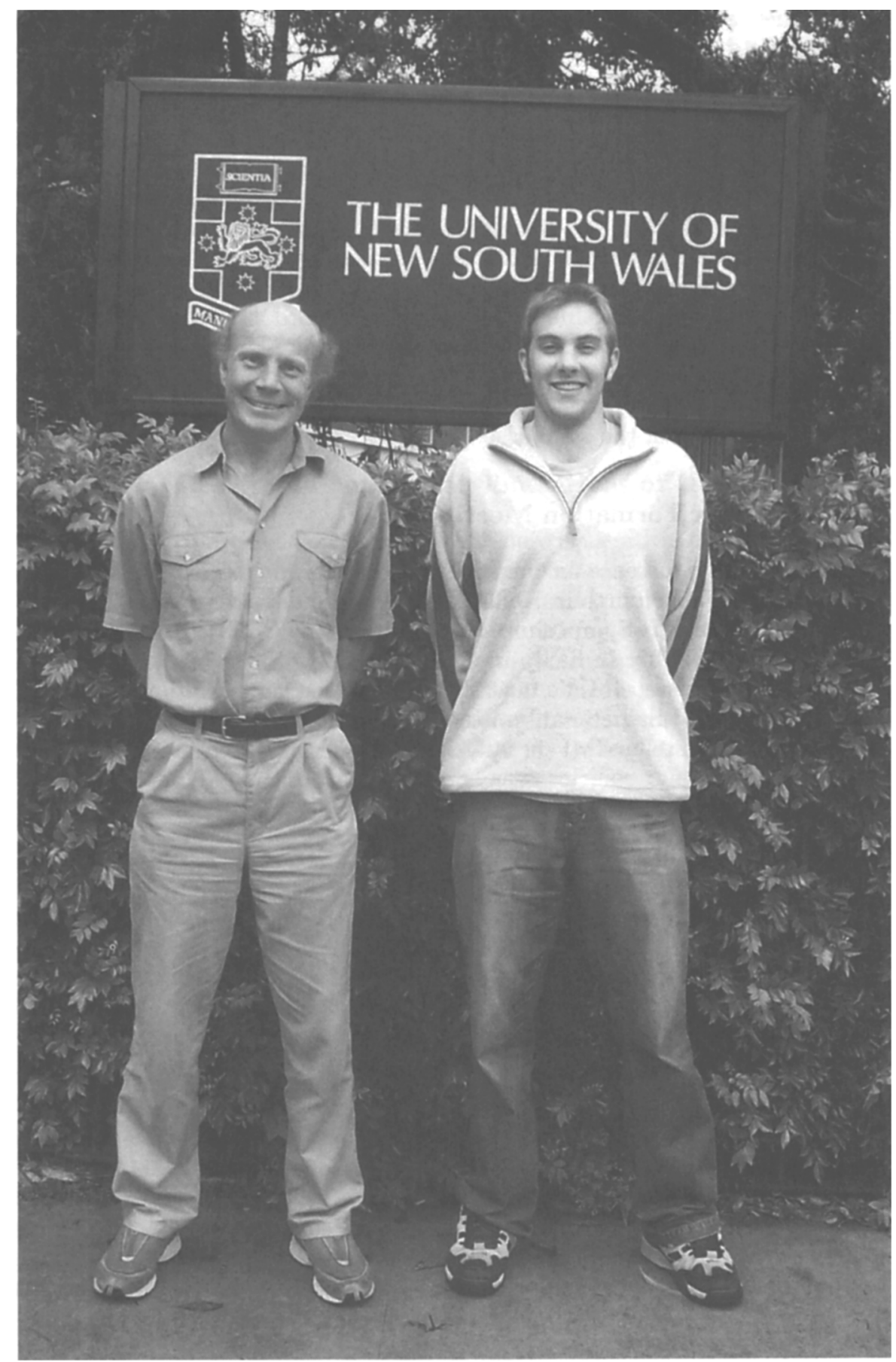

A happy Michael Burton and Steven Longmore after finishing these proceedings 